\title{
Intermittent Administration of Human Parathyroid Hormone before Osteosynthesis Stimulates Cancellous Bone Union in Ovariectomized Rats
}

\author{
Hiroyuki Tsuchie, ${ }^{1}$ Naohisa Miyakoshi, ${ }^{1}$ Yuji Kasukawa, ${ }^{1}$ Hiroshi Aonuma ${ }^{1}$ and \\ Yoichi Shimada ${ }^{1}$
}

${ }^{1}$ Department of Orthopedic Surgery, Akita University Graduate School of Medicine, Akita City, Akita, Japan

\begin{abstract}
It has been reported that intermittent administration of human parathyroid hormone (h-PTH) promotes bone healing after surgery for osteoporotic fractures. If bone healing is promoted by the administration of h-PTH during pre-operative waiting period, we can prevent prolonged bed rest. Therefore, we evaluated the effects of pre-operative h-PTH treatment on cancellous bone union and its mechanism for fracture healing in ovariectomized rats as a model for osteoporosis. Ovariectomized 7-month-old female Sprague-Dawley rats underwent an osteotomy of the proximal tibia as a fracture model, and h-PTH ( $30 \mu \mathrm{g} / \mathrm{kg}$ body weight) or vehicle was administered as a pre-operative treatment for one week. After the one-week treatment, tibiae were fixed with wire for osteosynthesis, and h-PTH or vehicle was administered for 1 or 3 weeks following wire fixation. In addition to bone histomorphometry, we used alcian blue/hematoxylin stained sections for evaluating cartilage volume and immunostained sections for analyzing the expression of proliferating cell nuclear antigen (PCNA) for cell proliferation and that of Sox9 and Runx2, differentiation markers for cartilage cells and osteoblasts, respectively. Pre-operative treatment with PTH significantly increased bone volume. Pre-operative and pre- to post-operative treatment with PTH for 2 weeks significantly promoted bone union. Pre-operative treatment with PTH significantly increased cartilage volume, and pre- to post-operative treatment with PTH for 2 weeks significantly increased the percentage of cells positive for Runx2 ( $p<0.01)$, but not PCNA or Sox9. Pre-operative administration of h-PTH enhances bone union by promoting cartilage formation and cell differentiation to osteoblasts, but not by promoting cell proliferation.
\end{abstract}

Keywords: cancellous bone union; intermittent administration; osteoporosis; parathyroid hormone; pre-operative period

Tohoku J. Exp. Med., 2013 Jan, 229 (1), 19-28. ㄷ 2013 Tohoku University Medical Press

Osteoporosis is characterized by low bone mass and microarchitectural deterioration of bone structures, resulting in bone fragility. Fall-related osteoporotic fractures are a serious medical problem in an aged society. Osteoporotic fractures often occur in cancellous bones, such as the femoral neck, distal radius, and vertebrae (Lemke 2005), and surgical treatment typically involves the use of screws or plates to stabilize the fracture site. Once fractures occur and osteosynthetic surgery is needed, careful management is required during surgery and the postoperative period as delayed bone formation or non-union of bone occurs in $6.2 \%$ to $22.0 \%$ of patients (Petersen et al. 1997; Findlay et al. 2002; Wenzl et al. 2004). Therefore, reductions in delayed bone formation or the non-union of bone after osteosynthetic surgery in osteoporotic patients is an important issue that needs to be resolved.

Intermittent administration of parathyroid hormone
(PTH) has been shown to have a potent anabolic effect on bone in various animal models and humans. PTH can increase bone mass in osteoporotic humans and rats with osteopenia induced by various causes (Tsuchida et al. 2000; Tsuchida et al. 2001; Kasukawa et al. 2004; Miyakoshi 2004). Several studies have reported that intermittent administration of human PTH also enhances fracture healing of cortical bones such as the shaft of the femur or tibia after surgical intervention in animals (Andeassen et al. 1999; Holzer et al. 1999; Nakazawa et al. 2005), even in an osteoporotic animal model (Jahng and Kim 2000). However, very few studies have addressed fracture healing of cancellous bones, which are frequent fracture sites in osteoporosis patients, after injury or surgical intervention (Komrakova et al. 2010). Therefore, a cancellous bone fracture model was created and an osteotomy of the proximal tibia was performed. The effects of intermittent admin-

Received August 8, 2012; accepted November 21, 2012. Published online December 5, 2012; doi: 10.1620/tjem.229.19.

Correspondence: Hiroyuki Tsuchie, Department of Orthopedic Surgery, Akita University Graduate School of Medicine, 1-1-1 Hondo, Akita, Akita 010-8543, Japan.

e-mail: tuchikiti@yahoo.co.jp 
istration of PTH on fracture healing were evaluated in this model based on a previous study (Nozaka et al. 2008).

However, surgical treatment is occasionally delayed in elderly patients because of medical complications that need to be monitored and the cessation of anticoagulants. In these cases, disadvantages exist because of prolonged bed rest, such as decreases in muscle volume, the deterioration of dementia, and pneumonia. An increase in the pre-operative period declines post-operative function and aggravates mortality (Hamlet et al. 1997; Chudyk et al. 2009). If administrating PTH from the preoperative waiting time promotes postoperative bone union, these disadvantages can be ameliorated. However, the effect of preoperative treatment has not been previously reported.

The aim of this study was to evaluate the effects of preoperative $\mathrm{PTH}$ treatment on cancellous bone union after an osteotomy of the proximal tibia in ovariectomized rats. Furthermore, to elucidate the mechanisms of cancellous bone healing induced by PTH, differentiation and proliferation of osteoblastic or chondroblastic cells were evaluated at the osteotomy site.

\section{Materials and Methods}

Animals

Seven-month-old female Sprague Dawley rats (Charles River Laboratory Inc., Kanagawa, Japan) were housed in a controlled environment at $22^{\circ} \mathrm{C}$ with a 12 -h light/dark cycle. Rats were pair fed and allowed free access to water and standard food (CE-2, Clea Japan Inc., Tokyo, Japan) containing $1.14 \%$ calcium, $1.06 \%$ phosphorus, and $250 \mathrm{IU}$ vitamin $\mathrm{D}_{3}$ per $100 \mathrm{~g}$ of food, as described previously (Tamura et al. 2001). Osteopenia was induced by a bilateral ovariectomy (OVX), as described previously (Abe et al. 1999). The response to OVX was confirmed by uterine atrophy when animals were sacrificed (Kim et al. 2002).

\section{Cancellous bone osteotomy at the proximal tibia}

A cancellous bone osteotomy of the right proximal tibia was performed, as previously described (Nozaka et al. 2008; Tsuchie et al. 2011), 4 weeks after a bilateral OVX, a time period that ensures sufficient osteopenia (Nitta et al. 1999). A median parapatellar incision from the knee joint through the proximal half of the tibia was made in the right hind limb, and a complete mid-sagittal osteotomy from the joint surface to the outer tibial diaphysis was performed using an electrically powered bone saw under general anesthesia with ketamine (Sankyo, Tokyo, Japan) and xylazine (Zenoaq, Fukushima, Japan) (Fig. 1a, b). To decrease pain after osteotomy, we used buprenorphine (Otsuka, Tokyo, Japan) for only one day after osteotomy and meloxicam (Kyoritsu, Tokyo, Japan) from two days after osteotomy. The osteotomized proximal tibia was fixed with circumferential wire $(0.4 \mathrm{~mm}$ diameter $) 1$ week after the osteotomy as an operation for cancellous bone fractures under general anesthesia (Fig. 1c). After osteosynthesis, animals were allowed to move freely. No animal had an abnormal gait or impaired locomotion postoperatively.

\section{Experimental design}

Animals were divided into the following 6 groups: (1) preoperative vehicle treated group (V group, $n=10$ ); (2) preoperative PTH treated group (P group, $n=10$ ); (3) preoperative vehicle and postop-

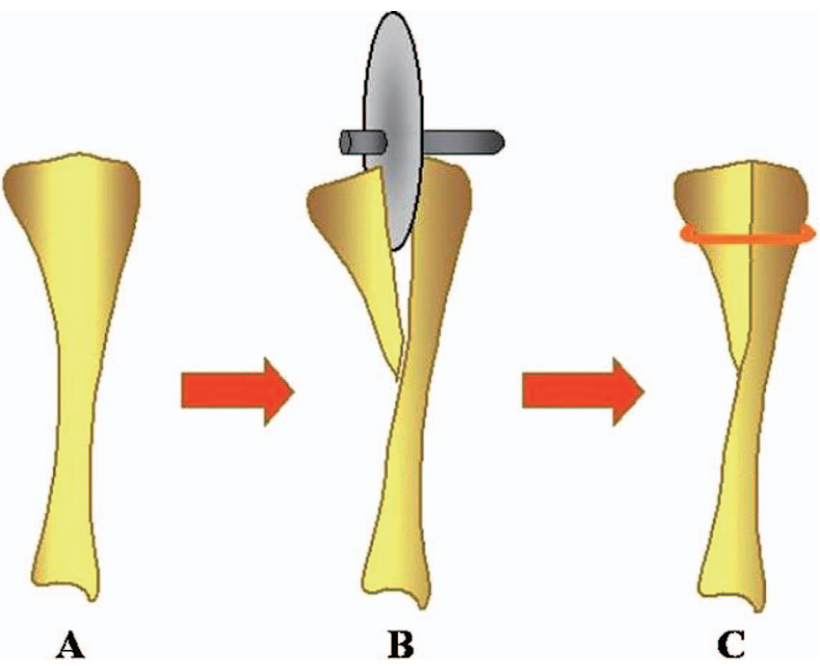

Fig. 1. Schema of the surgical procedure of the cancellous bone osteotomy.

A complete mid-sagittal osteotomy from the knee joint surface to the tibial outside diaphysis was performed using an electric bone saw (a, b). The osteotomized proximal tibia was then fixed with circumferential wire (0.4 mm diameter) 1 week after the osteotomy in all animals (c).

erative vehicle treated group (V-V group, $n=22)$; (4) preoperative vehicle and postoperative PTH treated group (V-P group, $n=21)$; (5) preoperative $\mathrm{PTH}$ and postoperative vehicle treated group $(\mathrm{P}-\mathrm{V}$ group, $n=21$ ); and (6) preoperative $\mathrm{PTH}$ and postoperative $\mathrm{PTH}$ treated group (P-P group, $n=21$ ). Human PTH [h-PTH (1-34), Asahi Kasei Pharma Co. Ltd., Tokyo, Japan] was dissolved in $0.1 \mathrm{M}$ sodium acetate buffer (PH 7.2) containing $0.1 \%$ bovine serum albumin (Seto et al. 1999). There is 4 amino-acid differences compared to rat PTH, but the activity or reaction of h-PTH to rat tissue are not different compared to those of rat PTH (Orloff JJ et al. 1991). h-PTH (30 $\mu \mathrm{g}$ / $\mathrm{kg}$ body weight) or its vehicle was administered subcutaneously 3 times a week for 2 or 4 weeks based on previous studies (Skripitz and Aspenberg 2001; Komatsubara et al. 2005) (Fig. 2).

Animals were euthanized under anesthesia with an intraabdominal injection of ketamine (Sankyo, Tokyo, Japan) and xylazine (Zenoaq, Fukushima, Japan). The V or P group was euthanized one week after the osteotomy, and the other groups were euthanized at two different time points of 2 and 4 weeks after the osteotomy. After bilateral tibiae were harvested, the bone mineral density (BMD) of all proximal tibiae was measured in $2.5 \mathrm{~mm}$ regions of the proximal tibia by dual energy X-ray absorptiometry (QDR-4500, Hologic Inc. Waltham, MA, USA). Thereafter, right tibiae were fixed in neutralbuffered formalin until being prepared for histological examination. Animal experimental protocols were approved by the Animal Committee of Akita University Graduate School of Medicine, and all animal experiments conformed to the "Guidelines for Animal Experimentation" of Akita University.

\section{Sample preparation}

The right proximal half of tibiae, including osteotomy sites, were decalcified with neutral $10 \%$ ethylene diamine tetra-acetic acid (EDTA) for 3 weeks and were embedded in paraffin. Five serial 3- $\mu$ m-thick mid-frontal sections were prepared for hematoxylin and eosin (H \& E) staining for cancellous bone histomorphometry, for 


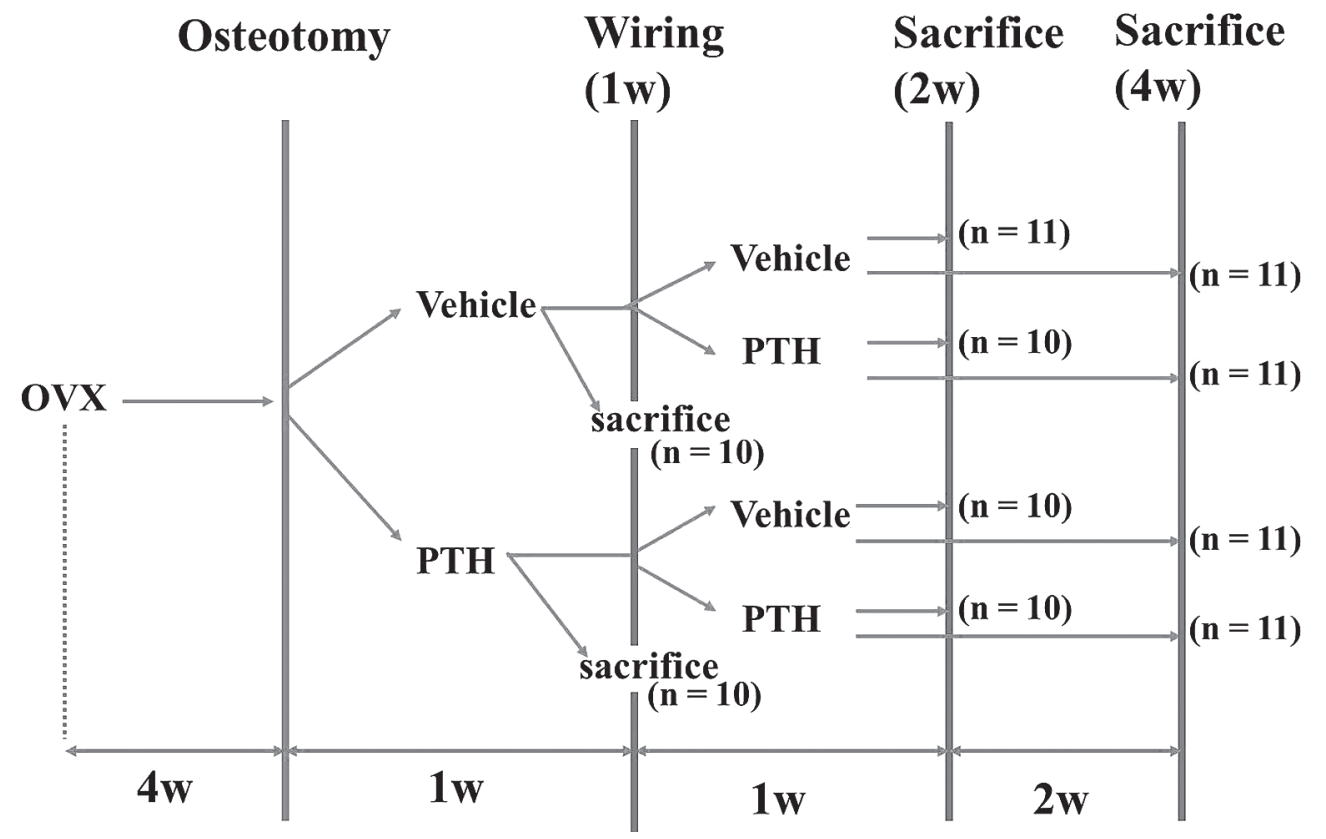

Fig. 2. Experimental groups and time schedule.

Animals were divided into the following 6 groups: (1) preoperative vehicle treated group (V group, $n=10$ ); (2) preoperative PTH treated group (P group, $n=10$ ); (3) preoperative vehicle and postoperative vehicle treated group (V-V group, $n=22$ ); (4) preoperative vehicle and postoperative PTH treated group (V-P group, $n=21$ ); (5) preoperative PTH and postoperative vehicle treated group (VP-V group, $n=21$ ); and (6) preoperative PTH and postoperative PTH treated group (P-P group, $n=21$ ). The $\mathrm{V}$ or $\mathrm{P}$ group was euthanized one week after the osteotomy, and the other groups were euthanized at two different time points of 2 and 4 weeks after the osteotomy.

alcian blue and hematoxylin staining for cartilage evaluation, and for immunohistochemical staining of proliferating cell nuclear antigen (PCNA), Sox9, and Runx2 to evaluate cell proliferation, differentiation to cartilage cells and differentiation to osteoblasts, respectively. We made all staining sections ( $\mathrm{H} \& \mathrm{E}$, alcian blue and hematoxylin, PCNA, Sox9, and Rux2) from all rat tibiae ( $n=10$ or 11 each group).

\section{Bone histomorphometry}

Bone histomorphometrical analyses with H-E-stained sections of the right osteotomy tibia were performed with a semiautomatic graphic system (Histometry RT Camera, System Supply Co., Nagano, Japan). Measurements were obtained in regions $390 \mu \mathrm{m}$ from the lowest point of growth plate-metaphyseal junctions in the caudal direction, to exclude the primary spongiosa, as well as $390 \mu \mathrm{m}$ from endocortical surfaces, at a magnification of $200 \times$ (Nakajima et al. 2001). The following histomorphometric parameters were measured, as described previously: cancellous bone volume per tissue volume (BV/TV), osteoid surface per bone surface (OS/BS), eroded surface (ES/BS), osteoclast surface (Oc.S/BS), and osteoclast number (Oc.N/ BS) (Parfitt et al. 1987).

\section{Evaluation of bone union after the osteotomy}

$\mathrm{H} \& \mathrm{E}$ stained sections obtained from each osteotomized tibia were used to evaluate cancellous bone union. The percentage of bone union (rate of the length of bone union to the total length of the osteotomy) was calculated with a semiautomatic graphic system (Histometry RT Camera, System Supply Co., Nagano, Japan) at a magnification of $100 \times$. Complete bone union (bone-to-bone binding at the osteotomy site) was defined as bone union, whereas fibrous union (fibrous binding) was defined as non-union (Nozaka et al. 2008).

\section{Analysis of cartilage in the callus}

Alcian blue and hematoxylin stained sections obtained from each osteotomized tibia, as described previously, were used to evaluate cartilage in the callus (Zhang et al. 2002). Measurements were obtained $390 \mu \mathrm{m}$ distal from the lowest point of growth plate-metaphyseal junctions to the crossing of the site at the endosteal surface, and the percentage of cartilage (the rate of cartilage to the total volume) was calculated with a semiautomatic graphic system (Histometry RT Camera, System Supply Co., Nagano, Japan) at a magnification of $100 \times$.

\section{Immunohistochemistry}

Other sets of sections of proximal tibiae, including the osteotomy site from each animal, were immunostained with a monoclonal antibody against PCNA (PC10, DAKO, Glostrup, Denmark) to evaluate cell proliferation activity, with a rabbit polyclonal antibody against Sox9 (H-90, Santa Cruz Biotechnology, Inc., Santa Cruz, USA) to evaluate differentiation to cartilage cells, and with a primary monoclonal antibody against Runx2 (MBL Co., Ltd., Tokyo, Japan) to evaluate differentiation to osteoblasts, as described previously (Meunier et al. 1971; Kamo et al. 2009; Konishi et al. 2010). Four randomized regions of interest (ROI) (size, $250 \mu \mathrm{m} \times 370 \mu \mathrm{m}$ each) were analyzed at the osteotomy site in the right proximal tibia, which corresponded to the measurement area for bone histomorphometry. The proportion of PCNA-, Sox9-, and Runx2-positive cells was calculated and expressed as a percentage of total cells. Averaged data 
obtained from four ROIs in each specimen was used for comparisons.

\section{Statistical analysis}

All values are expressed as the mean \pm standard deviation (S.D.). A Student $t$-test and Wilcoxon rank-sum test was used for comparisons between the vehicle and PTH groups at 1 week. A two-way analysis of variance (ANOVA) was used to test for significance. Differences among the four groups at 2 and 4 weeks, and among three time points in each of the four groups, were compared using the Scheffé test or Friedman test for multiple comparisons. Probability (P) values less than 0.05 were considered significant. All statistical analyses were performed using the Statistical Package for the Biosciences (SPBS V9.5).

\section{Results}

$B M D$

Though there was no significant increase in BMD in bilateral tibiae 1 or 2 weeks after PTH or Vehicle treatment, $\mathrm{BMD}$ of the right tibia in P-P and V-P groups were significantly greater than that of the $\mathrm{V}-\mathrm{V}$ group at 4 weeks $(p<$ 0.01 , Table 1). BMD in the P-P group at 4 weeks was significantly greater than that of the P-V group $(p<0.05)$.

In the left tibia (not osteotomized), only BMD in the P-P group was significantly greater than that of $\mathrm{V}-\mathrm{V}$ and $\mathrm{P}-\mathrm{V}$ groups ( $p<0.001$ and 0.01 , respectively) at 4 weeks.

\section{Bone histomorphometry}

$\mathrm{BV} / \mathrm{TV}$ and $\mathrm{OS} / \mathrm{BS}$ in the P-P group at 2 and 4 weeks were significantly greater than those of the $\mathrm{V}-\mathrm{V}$ group $(p<$ 0.01 , respectively, Table 2). BV/TV and OS/BS in the $\mathrm{P}-\mathrm{V}$ group were significantly lower than those of the P-P group $(p<0.05$ and $p<0.01$, respectively) at 4 weeks. Only BV/ $\mathrm{TV}$ in the V-P group was significantly greater than that of the $\mathrm{V}-\mathrm{V}$ group at 4 weeks $(p<0.05)$. OS/BS in the $\mathrm{P}$ and $\mathrm{V}-\mathrm{P}$ groups were significantly greater than those of $\mathrm{V}$ and $\mathrm{V}-\mathrm{V}$ groups at 1,2 , and 4 weeks $(p<0.01)$. OS/BS in the
$\mathrm{P}-\mathrm{V}$ group was significantly lower than that of the V-P group $(p<0.01)$ at 2 and 4 weeks.

However, bone resorption parameters, including ES/ BS, Oc.S/BS, and Oc.N/BS in P, V-P, and P-P groups were significantly lower than those in the $\mathrm{V}-\mathrm{V}$ group at all time points $(p<0.01)$, but these parameters immediately recovered to the levels of vehicle treated controls after the cessation of PTH treatment.

\section{Evaluation of Bone Union}

One week after the osteotomy, cancellous bone repair by new cartilage and woven bone was clearer in the P group (Fig. 3b) than that in the $\mathrm{V}$ group (Fig. 3a). Two weeks after the osteotomy, the P-P group (Fig. 3d) showed more remodeled cancellous bones at the osteotomy site than that of the V-V group (Fig. 3c), which revealed fibrous tissue at the osteotomy site. Bone union was almost completed in both P-P (Fig. 3f) and V-V groups (Fig. 3e). The trabecular thickness of remodeled cancellous bone in the P-P group (Fig. 3f) was thicker than that in the V-V group (Fig. 3e).

PTH treatment significantly increased cancellous bone union 1 week after the osteotomy, where the circumferential wire was not yet fixed $(p<0.05)$. Bone union 2 weeks after the osteotomy in P-P and P-V groups was significantly more advanced than that of V-V and V-P groups $(p<0.05)$. No significant differences in bone union among the groups were observed 4 weeks after the osteotomy (Table 3).

\section{Analysis of Cartilage}

PTH treatment significantly increased cartilage tissue 1 week after the osteotomy (Table 4$)$, where the circumferential wire was not yet fixed $(p<0.01)$ (Fig. 4). Cartilage tissue in the P-P group 2 and 4 weeks after the osteotomy was significantly larger than that of $\mathrm{V}-\mathrm{V}$ and $\mathrm{V}-\mathrm{P}$ groups ( $p<0.01$ and $p<0.05$ ). Two weeks after the osteotomy,

Table 1. PTH effects on the bone mineral density (BMD) of bilateral proximal tibiae.

\begin{tabular}{lccc}
\hline & & Right tibia $\left(\mathrm{g} / \mathrm{cm}^{2}\right)$ & Left tibia $\left(\mathrm{g} / \mathrm{cm}^{2}\right)$ \\
\hline Vehicle & $1 \mathrm{w}$ & $0.255 \pm 0.019$ & $0.276 \pm 0.015$ \\
PTH & $1 \mathrm{w}$ & $0.259 \pm 0.021$ & $0.286 \pm 0.018$ \\
Vehicle-Vehicle & $2 \mathrm{w}$ & $0.264 \pm 0.016$ & $0.279 \pm 0.016$ \\
Vehicle-PTH & $2 \mathrm{w}$ & $0.270 \pm 0.010$ & $0.277 \pm 0.012$ \\
PTH-Vehicle & 2w & $0.275 \pm 0.034$ & $0.277 \pm 0.018$ \\
PTH-PTH & 2w & $0.269 \pm 0.018$ & $0.290 \pm 0.013$ \\
Vehicle-Vehicle & $4 \mathrm{w}$ & $0.251 \pm 0.014$ & $0.269 \pm 0.017$ \\
Vehicle-PTH & $4 \mathrm{w}$ & $0.289 \pm 0.030^{\mathrm{a}}$ & $0.287 \pm 0.019$ \\
PTH-Vehicle & $4 \mathrm{w}$ & $0.261 \pm 0.023$ & $0.280 \pm 0.011$ \\
PTH-PTH & $4 \mathrm{w}$ & $0.292 \pm 0.022^{\mathrm{a}, \mathrm{b}}$ & $0.307 \pm 0.015^{\mathrm{c}, \mathrm{d}}$ \\
\hline
\end{tabular}

w: week.

All values are mean \pm standard deviation.

${ }^{a} P<0.01$ (Scheffé-test) vs. the Vehicle-Vehicle $4 \mathrm{w}$ group of the right tibia.

${ }^{\mathrm{b}} P<0.05$ (Scheffé-test) vs. the PTH-Vehicle $4 \mathrm{w}$ group of the right tibia.

c $P<0.01$ (Scheffé-test) vs. the Vehicle-Vehicle $4 \mathrm{w}$ group of the left tibia.

${ }^{\mathrm{d}} P<0.01$ (Scheffé-test) vs. the PTH-Vehicle $4 \mathrm{w}$ group of the left tibia. 
Table 2. PTH effects on bone volume and bone metabolism at the cancellous osteotomy site.

\begin{tabular}{lllllll}
\hline & & BV/TV $(\%)$ & OS/BS $(\%)$ & ES/BS (\%) & Oc.S/BS (\%) & Oc.N/BS (N/mm) \\
\hline Vehicle & $1 \mathrm{w}$ & $21.1 \pm 8.0$ & $9.5 \pm 4.1$ & $31.4 \pm 6.3$ & $8.6 \pm 2.4$ & $0.48 \pm 0.15$ \\
PTH & $1 \mathrm{w}$ & $23.7 \pm 4.8$ & $18.3 \pm 2.8^{\mathrm{a}}$ & $20.3 \pm 7.7^{\mathrm{a}}$ & $3.7 \pm 1.8^{\mathrm{a}}$ & $0.22 \pm 0.10^{\mathrm{a}}$ \\
Vehicle-Vehicle & 2w & $18.8 \pm 3.6$ & $15.0 \pm 3.8$ & $36.5 \pm 6.0$ & $9.4 \pm 2.6$ & $0.48 \pm 0.12$ \\
Vehicle-PTH & 2w & $22.8 \pm 5.2$ & $31.2 \pm 2.9^{\mathrm{b}, \mathrm{c}}$ & $26.3 \pm 5.5^{\mathrm{b}, \mathrm{c}}$ & $5.3 \pm 1.5^{\mathrm{b}, \mathrm{c}}$ & $0.30 \pm 0.08^{\mathrm{b}, \mathrm{c}}$ \\
PTH-Vehicle & 2w & $24.4 \pm 5.2^{\mathrm{b}^{\prime}}$ & $16.7 \pm 5.4$ & $35.0 \pm 4.2$ & $8.6 \pm 1.7$ & $0.45 \pm 0.08$ \\
PTH-PTH & 2w & $29.1 \pm 6.5^{\mathrm{b}}$ & $31.2 \pm 5.8^{\mathrm{b}, \mathrm{c}}$ & $24.2 \pm 6.3^{\mathrm{b}, \mathrm{c}}$ & $5.0 \pm 1.1^{\mathrm{b}, \mathrm{c}}$ & $0.27 \pm 0.05^{\mathrm{b}, \mathrm{c}}$ \\
Vehicle-Vehicle & 4w & $15.5 \pm 4.6$ & $15.9 \pm 2.1$ & $35.0 \pm 8.2$ & $9.9 \pm 2.4$ & $0.55 \pm 0.12$ \\
Vehicle-PTH & 4w & $25.6 \pm 8.4^{\mathrm{d}^{\prime}}$ & $30.4 \pm 4.9^{\mathrm{d}, \mathrm{e}}$ & $24.0 \pm 5.8^{\mathrm{d}^{\prime}, \mathrm{e}}$ & $4.4 \pm 1.5^{\mathrm{d}, \mathrm{e}}$ & $0.27 \pm 0.10^{\mathrm{d}, \mathrm{e}}$ \\
PTH-Vehicle & $4 \mathrm{w}$ & $18.4 \pm 8.5$ & $19.0 \pm 5.3$ & $36.3 \pm 6.5$ & $8.8 \pm 1.5$ & $0.50 \pm 0.12$ \\
PTH-PTH & $4 \mathrm{w}$ & $29.6 \pm 6.9^{\mathrm{d}, \mathrm{e}^{\prime}}$ & $31.5 \pm 8.5^{\mathrm{d}, \mathrm{e}}$ & $22.1 \pm 4.1^{\mathrm{d}, \mathrm{e}}$ & $4.5 \pm 1.3^{\mathrm{d}, \mathrm{e}}$ & $0.29 \pm 0.11^{\mathrm{d}, \mathrm{e}}$ \\
\hline
\end{tabular}

w: week.

All values are mean \pm standard deviation.

${ }^{a} P<0.01$ (Student $t$-test) vs. the Vehicle $1 \mathrm{w}$ group in each evaluation.

${ }^{\mathrm{b}} P<0.01$ and ${ }^{\mathrm{b}^{\prime}} P<0.05$ (Scheffé-test and Friedman test) vs. the Vehicle-Vehicle $2 \mathrm{w}$ group in each evaluation.

c $P<0.01$ (Scheffé-test) vs. the PTH-Vehicle $2 \mathrm{w}$ group in each evaluation.

${ }^{\mathrm{d}} P<0.01$ and ${ }^{\mathrm{d}} P<0.05$ (Scheffé-test and Friedman test) vs. the Vehicle-Vehicle $4 \mathrm{w}$ group in each evaluation.

${ }^{\mathrm{e}} P<0.01$ and ${ }^{\prime} P<0.05$ (Scheffé-test and Friedman test) vs. the PTH-Vehicle 4w group in each evaluation.
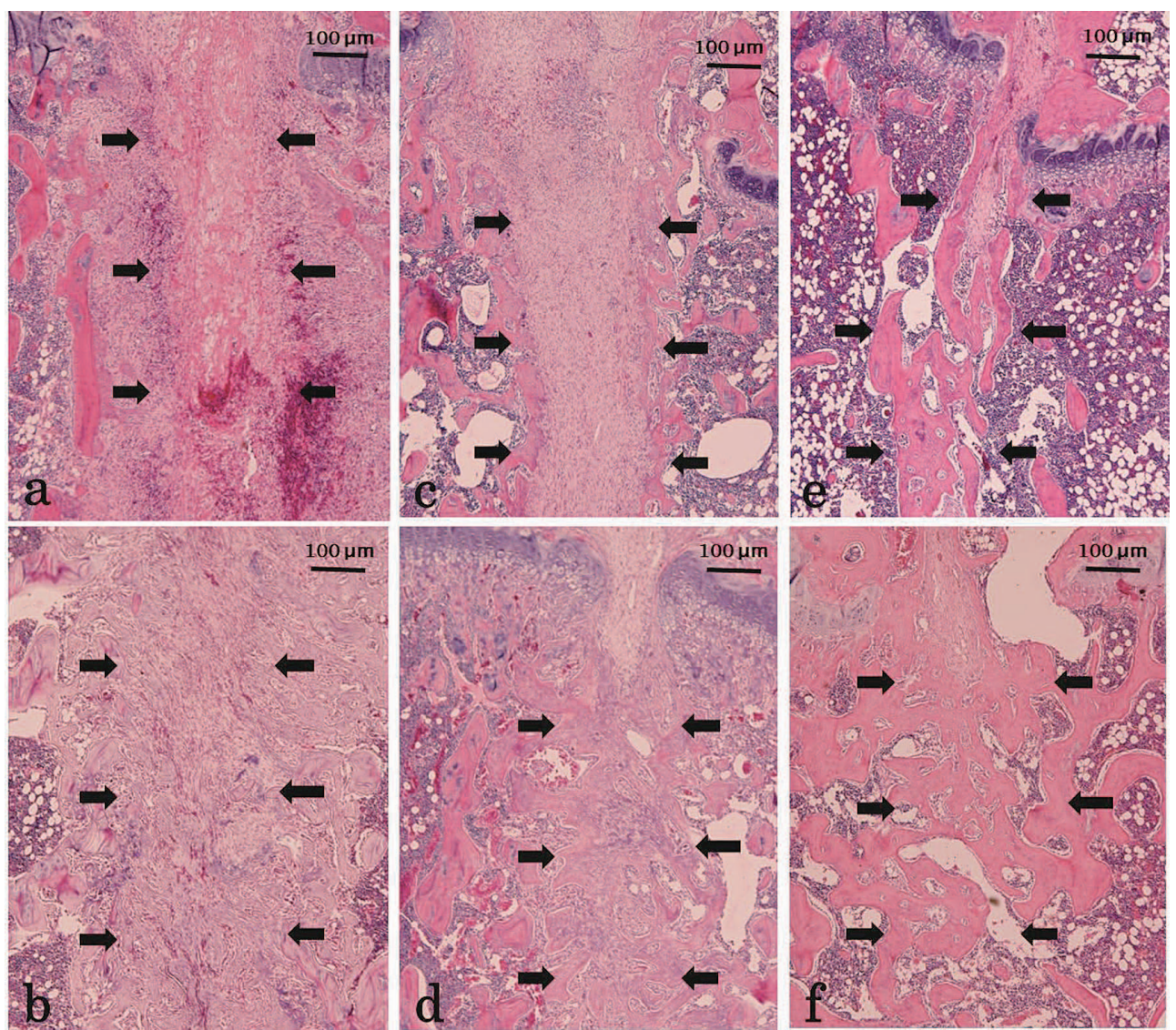

Fig. 3. Histological sections stained with hematoxylin and eosin at the osteotomy site.

Shown are the histological sections stained with hematoxylin and eosin at the osteotomy site with a magnification of $40 \times$. Osteotomy sites are showed by arrows. One week after the osteotomy, the amount of new cartilage and woven bone produced in the PTH group (b) was greater than that in the Vehicle group (a). Two weeks after the osteotomy, the amount of remodeled new cancellous bones observed at the osteotomy site in the PTH-PTH group (d) was greater than that in the Vehicle-Vehicle group (c). Four weeks after the osteotomy, bone union was almost finished in both the PTHPTH (f) group and Vehicle-Vehicle (e) group. 
Table 3. PTH effects on the bone union rate at the cancellous osteotomy site.

\begin{tabular}{llc}
\hline & & Bone union rate $(\%)$ \\
\hline Vehicle & $1 \mathrm{w}$ & 0 \\
PTH & $1 \mathrm{w}$ & ${\underline{8.5 \pm 13.3^{\mathrm{a}}}}^{5.9 \pm 10.1}$ \\
Vehicle-Vehicle & 2w & $4.1 \pm 8.6$ \\
Vehicle-PTH & 2w & $\underline{33.2 \pm 28.7^{\mathrm{bcc}}}$ \\
PTH-Vehicle & 2w & $\underline{24.6 \pm 18.2^{\mathrm{c}}}$ \\
PTH-PTH & 2w & $24.2 \pm 16.6$ \\
Vehicle-Vehicle & $4 \mathrm{w}$ & $23.8 \pm 18.1$ \\
Vehicle-PTH & $4 \mathrm{w}$ & $28.6 \pm 14.1$ \\
PTH-Vehicle & $4 \mathrm{w}$ & $31.5 \pm 17.9$ \\
PTH-PTH & $4 \mathrm{w}$ &
\end{tabular}

w: week

All values are mean \pm standard deviation

a $P<0.05$ (Wilcoxon rank-sum test) vs. the bone union rate of the Vehicle $1 \mathrm{w}$ group.

${ }^{\mathrm{b}} P<0.05$ (Friedman test) vs. the bone union rate of the Vehicle-Vehicle $2 \mathrm{w}$ group.

${ }^{c} P<0.05$ (Friedman test) vs. the bone union rate of the Vehicle-PTH $2 \mathrm{w}$ group.
Table 4. PTH effects on the percentage of cartilage tissue.

\begin{tabular}{lcc}
\hline & & Percentage of Cartilage (\%) \\
\hline Vehicle & 1w & $7.5 \pm 3.8$ \\
PTH & 1w & $\underline{15.5 \pm 6.8^{\mathrm{a}}}$ \\
Vehicle-Vehicle & 2w & $4.8 \pm 2.2$ \\
Vehicle-PTH & 2w & $5.2 \pm 3.2$ \\
PTH-Vehicle & 2w & ${\underline{8.6 \pm 4.4^{\mathrm{b}}}}^{\mathrm{10}}$ \\
PTH-PTH & 2w & $1.2 \pm 3.9^{\mathrm{b}^{\prime} \mathrm{c}}$ \\
Vehicle-Vehicle & $4 \mathrm{w}$ & $1.2 \pm 1.2$ \\
Vehicle-PTH & 4w & $1.7 \pm 2.3$ \\
PTH-Vehicle & $4 \mathrm{w}$ & $2.9 \pm 2.5^{\mathrm{d}, \mathrm{e}}$ \\
PTH-PTH & $4 \mathrm{w}$ &
\end{tabular}

w: week.

All values are mean \pm standard deviation.

a $P<0.01$ (Wilcoxon rank-sum test) vs. the Vehicle $1 \mathrm{w}$ group.

${ }^{\mathrm{b}} P<0.05$ and ${ }^{\mathrm{b}} P<0.01$ (Scheffé-test) vs. the Vehicle-

Vehicle $2 \mathrm{w}$ group.

c $P<0.05$ (Scheffé-test) vs. the Vehicle-PTH $2 \mathrm{w}$ group.

d $P<0.01$ (Friedman test) vs. the Vehicle-Vehicle $4 \mathrm{w}$ group.

${ }^{\mathrm{e}} P<0.05$ (Friedman test) vs. the Vehicle-PTH $4 \mathrm{w}$ group.
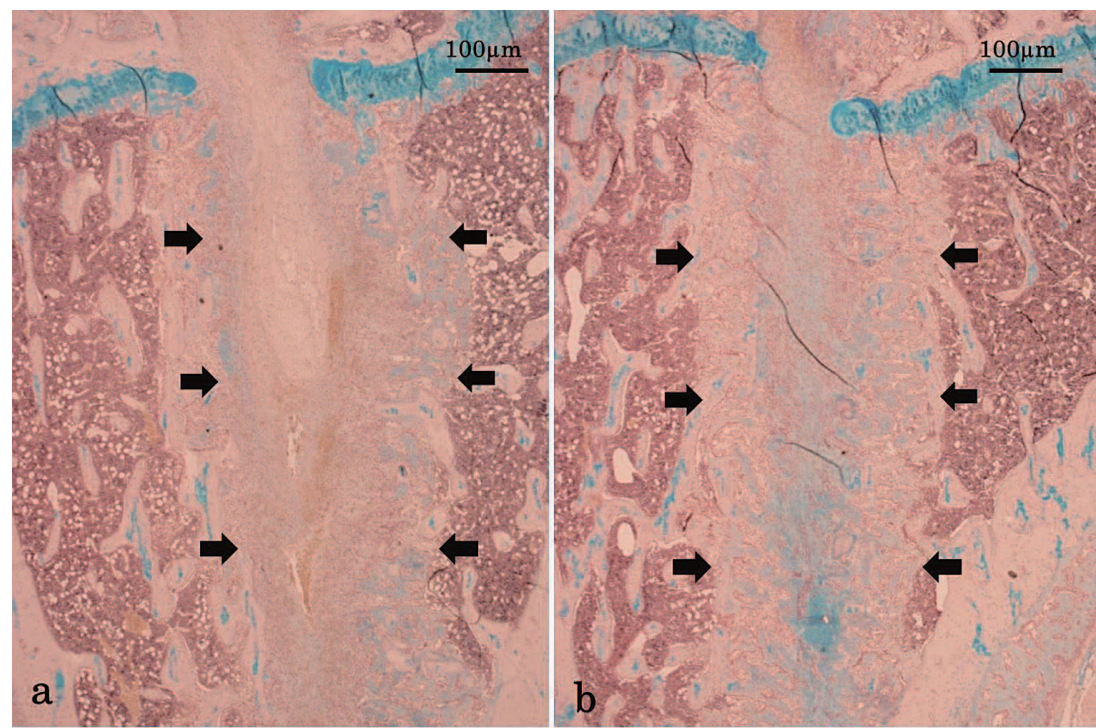

Fig. 4. Histological sections stained with alcian blue/hematoxylin at the osteotomy site.

Shown are the histological sections stained with alcian blue/hematoxylin ( $a$ and $b$ ) with a magnification of $20 \times$. Osteotomy sites are showed by arrows. Cartilage tissue is stained blue. One week after the osteotomy, the amount of new cartilage produced in the PTH group (b) was greater than that in the Vehicle group (a).

cartilage tissue of the $\mathrm{P}-\mathrm{V}$ group was significantly larger than that of the $\mathrm{V}-\mathrm{V}$ group.

\section{Immunohistochemistry}

Although PTH treatment did not show any effect on PCNA at an early stage after the osteotomy (Table 5), the P-P group showed advanced expression of PCNA 4 weeks after the osteotomy $(p<0.05)$ (Fig. 5a, b). Some Sox9 positive cells were observed in the $\mathrm{P}$ group only 1 week after the osteotomy (Fig. 5d). However, there were only a few Sox9 positive cells in the P-P group at 4 weeks (Fig. 5c). There was no significant difference in Sox 9 among groups at all time points. The percentage of Runx2 positive cells in the P-P group 2 and 4 weeks after the osteotomy was significantly higher than that of the V-V group ( $p<$ 0.05 ) (Fig. 5e, f). Four weeks after the osteotomy, the num- 
Table 5. PTH effects on the percentage of immunohistochemistry.

\begin{tabular}{|c|c|c|c|c|}
\hline & & PCNA (\%) & Sox9 (\%) & Runx2 (\%) \\
\hline Vehicle & $1 \mathrm{w}$ & $16.2 \pm 12.8$ & $2.17 \pm 2.08$ & $1.5 \pm 3.1$ \\
\hline PTH & $1 \mathrm{w}$ & $16.5 \pm 7.8$ & $2.73 \pm 2.80$ & $3.1 \pm 3.6$ \\
\hline Vehicle-Vehicle & $2 w$ & $9.3 \pm 5.8$ & $0.70 \pm 0.61$ & $15.8 \pm 4.4$ \\
\hline Vehicle-PTH & $2 w$ & $11.7 \pm 9.8$ & $0.87 \pm 0.71$ & $22.9 \pm 6.4$ \\
\hline PTH-Vehicle & $2 w$ & $15.2 \pm 5.3$ & $0.61 \pm 0.43$ & $22.6 \pm 8.5$ \\
\hline РTH-РTH & $2 w$ & $15.6 \pm 6.9$ & $1.32 \pm 1.05$ & $28.1 \pm 6.5^{\mathrm{b}}$ \\
\hline Vehicle-Vehicle & $4 w$ & $7.8 \pm 2.8$ & $0.93 \pm 0.89$ & $9.2 \pm 4.7$ \\
\hline Vehicle-PTH & $4 w$ & $8.7 \pm 4.0$ & $0.64 \pm 0.59$ & $16.7 \pm 6.9^{c}$ \\
\hline PTH-Vehicle & $4 w$ & $8.8 \pm 3.7$ & $0.64 \pm 0.59$ & $10.9 \pm 6.9$ \\
\hline РТН-РТН & $4 w$ & $13.4 \pm 5.4^{\mathrm{a}}$ & $0.98 \pm 1.59$ & $16.7 \pm 6.5^{\mathrm{c}}$ \\
\hline
\end{tabular}

w: week.

All values are mean \pm standard deviation.

${ }^{a} P<0.05$ (Scheffé-test) vs. PCNA of the Vehicle-Vehicle 4w group.

${ }^{\mathrm{b}} P<0.05$ (Scheffé-test) vs. Runx 2 of the Vehicle-Vehicle $2 \mathrm{w}$ group.

${ }^{c} P<0.05$ (Scheffé-test) vs. Runx2 of the Vehicle-Vehicle 4w group.
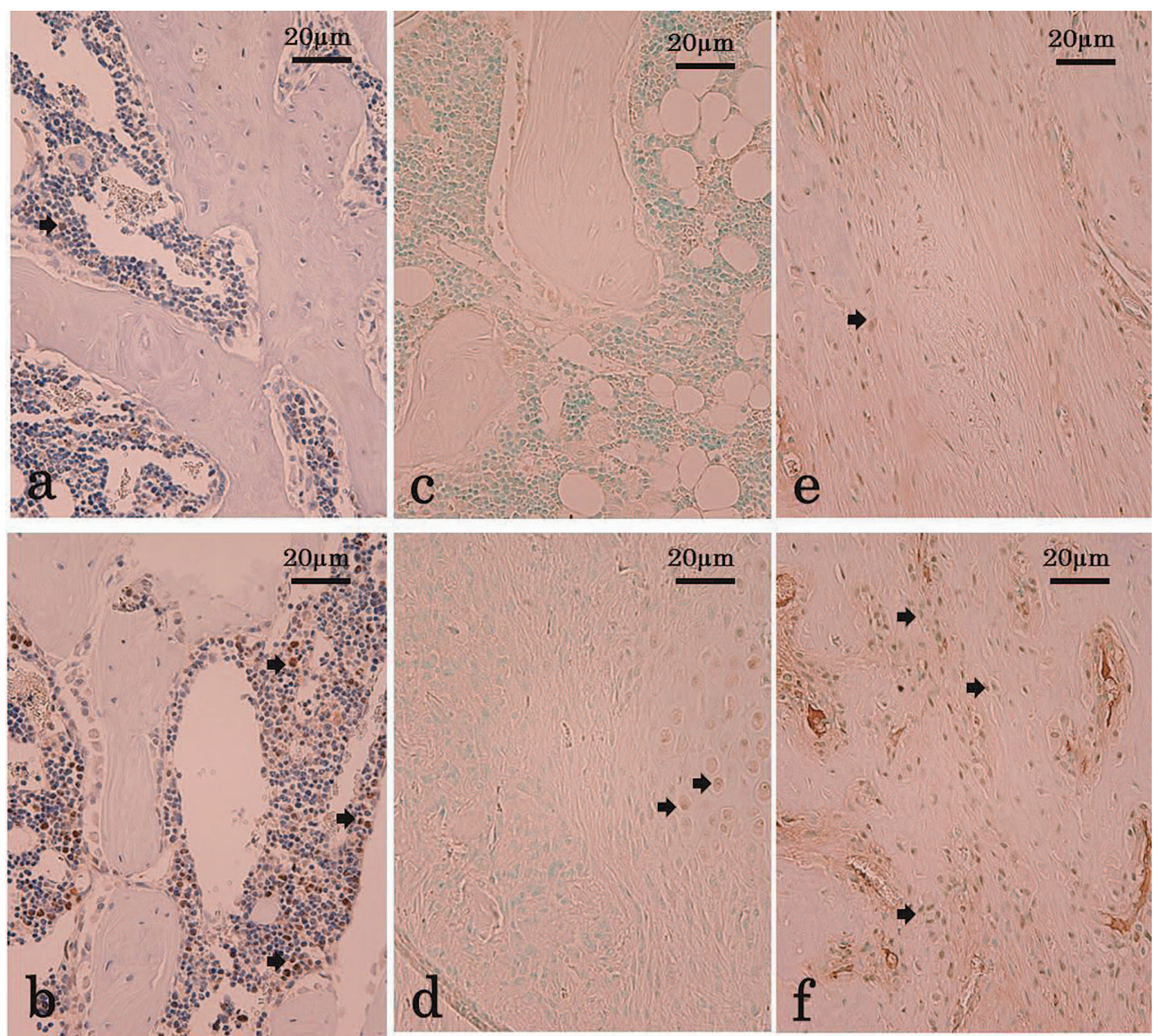

Fig. 5. Immunostaining for PCNA, Sox9, and Runx2 at the osteotomy site.

Shown are the sections immunostained with antibodies against PCNA (a and b), Sox9 (c and d), and Runx2 (e and f) at the osteotomy site with a magnification of $200 \times$. Immunostained positive cells stained dark reddish-brown dye (Some of them are showed by arrows). Four weeks after the osteotomy, PCNA positive cell numbers in the PTH-PTH group (b) were higher than those in the Vehicle-Vehicle (a) group. Some Sox9 positive cells were observed in the PTH group at 1 week (d), but there were hardly any Sox9 positive cells in the PTH-PTH group at 4 weeks (c). Two weeks after the osteotomy, Runx2 positive cell numbers in the PTH-PTH group (f) were higher than those in the Vehicle-Vehicle (e) group. 
ber of Runx2 positive cells of the V-P group was significantly higher than that of the $\mathrm{V}-\mathrm{V}$ group.

\section{Discussion}

Although the effect of advanced bone union by intermittent PTH administration in animal tests has been reported extensively, no studies examined the effect of administering PTH in the preoperative waiting period of osteosynthesis. It was previously reported that pretreatment with intermittent PTH administration was not effective for bone union (Komathubara et al. 2005). However, this study administered PTH before, not after a fracture occurred.

The present study evaluated the effects of PTH treatment on bone union in the period before osteosynthesis surgery after a fracture occurred. In our previous preliminary study, wiring surgery had significant effects on bone union after an osteotomy of the proximal tibia over that of sham surgery, with an obvious effect on cancellous bone union as an osteosynthesis surgery (Tsuchie et al. 2011).

In this study, pre-osteosynthesis PTH treatment, but not post-osteosynthesis, significantly advanced cancellous bone union 2 weeks after the osteotomy. As a result, intermittent PTH administration in the preoperative waiting period appears to be more important than in the postoperative period, and early administration after a fracture occurs is very important. In addition, PTH treatment advanced bone union 1 week after the osteotomy. At this time point, osteosynthesis was not conducted and there was a wide gap among bone fragments. Intermittent PTH administration may help fill in some gaps between fragments. This is advantageous for preventing pseudoarthrodesis, even in non-operative treatments. There was no significant difference in bone union 4 weeks after the osteotomy because most bone unions at osteotomy sites were finished in all groups. This measurement method of bone union does not show a high percentage of bone union because newly formed bone at the osteotomy site is absorbed and changed to bone marrow tissue (Tsuchie et al. 2011).

In bone histomorphometrical analyses, PTH administration induced increased bone formation and bone volume. Four weeks administration of PTH significantly increased BMD of both osteotomy and non-osteotomy sites, and postoperative treatment with PTH significantly increased BMD at the osteotomy site only. As such, intermittent administration of PTH before and after osteosynthesis may save cancellous bone at the bone union site. In this study, our PTH administration protocol increased bone formation and suppressed bone absorption. In recent years, some animal or human studies have reported that bone absorption was suppressed by intermittent administration of PTH (Nozaka et al. 2008). We may assume the deterioration of bone quality when we think about suppressed bone absorption. However, bone absorption was not suppressed more in the PTH treated OVX rat than that in the non-PTH treated nonOVX rat (Nozaka et al. 2008). Therefore, we can consider that intermittent administration of PTH for OVX rats sup- presses the redundant osteoclasts induced by OVX. This treatment is ideal for osteoporosis patients whose bone absorption is accelerated.

Intermittent administration of PTH promotes Runx2 expression and leads the differentiation from mesenchymal cells to osteoblasts, ultimately promoting bone formation (Tintut et al. 1999; Bellido et al. 2003; Krishnan et al. 2003). In this study, Runx 2 peaked 2 weeks after the osteotomy in all groups. The expression of Runx2 in the P-P group was significantly higher than that in the V-V group. Intermittent PTH administration promoted bone union by advancing Runx2 expression and the differentiation to osteoblasts from osteotomy site cells.

Regarding the effects of PTH effects on cancellous bone repair, cartilage tissue at the osteotomy site was significantly increased in the PTH treated group before wiring. Cancellous bone repair of the proximal tibia is caused by endochondral ossification (Tsuchie et al. 2011), and when cartilage tissues combine after wiring, it seems that bone union is accelerated more in the PTH treated group. Recently, some reports stated that the effect of accelerated bone union by intermittent PTH administration was related to the accelerated formation of cartilage tissue (Nakazawa et al. 2005; Kakar et al. 2007; Kaback et al. 2008). However, Sox9 expression, which is involved in the differentiation of cartilage tissue, was not accelerated at all time points. Sox9 is expressed in the precursor of chondrocytes and chondrocytes, but this expression dramatically decreases in hypertrophic chondrocytes (Akiyama et al. 2002). It was suggested that a peak in Sox 9 expression occurred at the osteotomy site 5 days after the osteotomy and rapidly declined at 7 days (Kakar et al. 2007). Because most cartilage tissues at the osteotomy site were not precursor chondrocytes and chondrocytes 1 week after the osteotomy, Sox 9 advanced expression may not have appeared in our study.

Although PCNA peaked 1 week after the osteotomy in all groups, PCNA expression 1 and 2 weeks after the osteotomy with intermittent PTH administration was not higher than that with the vehicle administration. These results show that PTH administration did not provoke cell proliferation at the osteotomy site during the healing course following a bone fracture. Based on these findings, intermittent PTH administration initially promoted differentiation from mesenchymal cells to chondrocytes, and then promoted differentiation from chondrocytes to osteoblasts. Although PTH enhances the general circulation of endochondral ossification at the osteotomy site, PTH did not cause cell proliferation.

Various causes of stimulating bone formation by PTH have been reported, such as the promotion of Runx2, insulin-like growth factor-1 (IGF-1), and activating protein-1 (AP-1), and the suppression of sclerostin and smad ubiquitin regulatory factor (smurf) (Tintut et al. 1999; Miyakoshi et al. 2001; Bellido et al. 2003; Krishnan et al. 2003; Qin et al. 2003; Sutherland et al. 2004). Although the involvement 
of the Wnt family has been reported (Kakar et al. 2007), the detailed mechanisms of bone union at the fracture site by PTH administration are still unclear. Further examinations are needed to clarify the mechanism of intermittent PTH administration on bone union.

In conclusion, the present study is the first to our knowledge to evaluate the pretreatment effect of PTH in a cancellous bone fracture model. Pre-operative intermittent administration of PTH enhances bone union in ovariectomized rats by promoting cartilage formation and cell differentiation to osteoblasts, but not by promoting cell proliferation. Since detailed mechanisms of stimulating bone union by PTH administration are still unclear, further examinations are needed to clarify these mechanisms for the treatment of human osteoporotic fractures.

\section{Acknowledgments}

The authors would like thank to Ms. Kaoru Sakamoto and Ms. Rie Kamoya for their technical assistance.

\section{Conflict of Interest}

The authors declare no conflict of interest.

\section{References}

Abe, T., Sato, K., Miyakoshi, N., Kudo, T., Tamura, Y., Tsuchida, T. \& Kasukawa, Y. (1999) Trabecular remodeling process in the ovariectomized rat: modified nodestrut analysis. Bone, 24, 591-596.

Akiyama, H., Chaboissier, M.C., Martin, J.F., Schedl, A. \& de Crombugghe, B. (2002) The transcription factor Sox9 has essential roles in successive steps of the chondrocyte differentiation pathway and is required for expression of Sox 5 and Sox6. Genes. Dev., 16, 2813-2828.

Andreassen, T.T., Ejersted, C. \& Oxlund, H. (1999) Intermittent parathyroid hormone (1-34) treatment increases callus formation and mechanical strength of healing rat fractures. J. Bone Miner. Res., 14, 960-968.

Bellido, T., Ali, A.A., Plotkin, L.I., Fu, Q., Gubrij, I., Roberson, P.K., Weinstein, R.S., O’Brien, C.A., Manolagas, S.C. \& Jilka, R.L. (2003) Proteasomal degradation of Runx2 shortens parathyroid hormone-induced anti-apoptotic signaling in osteoblasts. A putative explanation for why intermittent administration is needed for bone anabolism. J. Biol. Chem., 278, 50259-50272.

Chudyk, A.M., Jutai, J.W., Petrella, R.J. \& Speechley, M. (2009) Systemic review of hip fracture rehabilitation practices in the elderly. Arch. Phys. Med. Rehabil., 90, 246-262.

Findlay, S.C., Eastell, R. \& Ingle, B.M. (2002) Measurement of bone adjacent to tibial shaft fracture. Osteoporos. Int., 13, 980-989.

Hamlet, W.P., Lieberman, J.R., Freedman, E.L., Dorey, F.J., Fletcher, A. \& Johnson, E.E. (1997) Influence of health status and the timing of surgery on mortality in hip fracture patients. Am. J. Orthop., 26, 621-627.

Holzer, G., Majeska, R.J., Lundy, M.W., Hartke, J.R. \& Einhorn, T.A. (1999) Parathyroid hormone enhances fracture healing. A preliminary report. Clin. Orhop. Relat. Res., 366, 258-263.

Jahng, J.S. \& Kim, H.W. (2000) Effect of intermittent administration of parathyroid hormone on fracture healing in ovariectomized rats. Orthopedics, 23, 1089-1094.

Kaback, L.A., Soung, D.Y., Naik, A., Geneau, G., Schwarz, E.M., Rosier, R.N., O'Keefe, R.J. \& Drissi, H. (2008) Teriparatide (1-34 human PTH) regulation of osterix during fracture repair.
J. Cell Biochem., 105, 219-226.

Kakar, S., Einhorn, T.A., Vora, S., Miara, L.J., Hon, G., Wigner, N.A., Toben, D., Jacobsen, K.A., AI-Sebaei, M.O., Song, M., Trackman, P.C., Morgan, E.F., Gerstenfeld, L.C. \& Bames, G.L. (2007) Enhanced chondrogenesis and Wnt signaling in PTH-treated fractures. J. Bone Miner. Res., 22, 1903-1912.

Kamo, K., Miyakoshi, N., Kasukawa, Y., Sasaki, H. \& Shimada, Y. (2009) Effects of single and cyclical local injections of basic fibroblast growth factor on cancellous bone defects in rabbits. J. Orthop. Sci., 14, 811-819.

Kasukawa, Y., Miyakoshi, N., Itoi, E., Tsuchida, T., Tamura, Y., Kudo, T., Suzuki, K., Seki, A. \& Sato, K. (2004) Effects of h-PTH on cancellous bone mass, connectivity, and bone strength in ovariectomized rats with and without sciaticneurectomy. J. Orthop. Res., 22, 457-464.

Kim, H.J., Bae, Y.C., Park, R.W., Choi, S.W., Cho, S.H., Choi, Y.S. \& Lee, W.J. (2002) Bone-protecting effect of safflower seeds in ovariectomized rats. Calcif. Tissue Int., 71, 88-94.

Komatsubara, S., Mori, S., Mashiba, T., Nonaka, K., Aeki, A., Akiyama, T., Miyamoto, K., Cao, Y., Manabe, T. \& Norimatsu, H. (2005) Human parathyroid hormone (1-34) accelerates the fracture healing process of woven to lamellar bone replacement and new cortical shell formation in rat femora. Bone, 36, 678-687.

Komrakova, M., Stuermer, E.K., Werner, C., Wicke, M., Kolios, L., Sehmisch, S., Tezval, M., Daub, F., Martens, T., Witzenhausen, P., Dullin, C. \& Stuermer, K.M. (2010) Effect of human parathyroid hormone hPTH (1-34) applied at different regimes on fracture healing and muscle in ovariectomized and healthy rats. Bone, 47, 480-492.

Konishi, E., Nakashima, Y., Iwasa, Y., Nakao, R. \& Yanagizawa, A. (2010) Immunohistochemical analysis for Sox9 reveals the cartilaginous character of chondroblastoma and chondromyxoid fibroma of the bone. Hum. Pathol., 41, 208-213.

Krishnan, V., Moore, T.L., Ma, Y.L., Helvering, L.M., Frolik, C.A., Valasek, K.M., Ducy, P. \& Geiser, A.G. (2003) Parathyroid hormone bone anabolic action requires Cbfa1/Runx2-dependent signaling. Mol. Endocrinol., 17, 423-435.

Lemke, D.M. (2005) Vertebroplasty and kyphoplasty for treatment of painful osteoporotic compression fractures. J. Am. Acad. Nurse Pract., 17, 268-276.

Meunier, P., Aaron, J., Edouard, C. \& Vignon, G. (1971) Osteoporosis and the replacement of cell populations of the marrow by adipose tissue. A quantitative study of 84 iliac bone biopsies. Clin. Orthop. Relat. Res., 80, 147-154.

Miyakoshi, N. (2004) Effects of parathyroid hormone on cancellous bone mass and structure in osteoporosis. Curr. Pharm. Des., 10, 2615-2627.

Miyakoshi, N., Kasukawa, Y., Linkhart, T.A., Baylink, D.J. \& Mohan, S. (2001) Evidence that anabolic effects of PTH on bone require IGF-1 in growing mice. Endocrinology, 142, 4349-4356.

Nakajima, A., Nakajima, F., Shimizu, S., Ogasawara, A., Wanaka, A., Moriya, H., Einhorn, T.A. \& Yamazaki, M. (2001) Spatial and temporal gene expression for fibroblast growth factor type I receptor (FGFR1) during fracture healing in the rat. Bone, 29, 458-466.

Nakazawa, T., Nakajima, A., Shiomi, K., Moriya, H., Einhorn, T.A. \& Yamazaki, M. (2005) Effects of low-dose, intermittent treatment with recombinant human parathyroid hormone (1-34) on chondrogenesis in a model of experimental fracture healing. Bone, 37, 711-719.

Nitta, T., Fukushima, T., Nakamuta, H. \& Koida, M. (1999) Glucocorticoid-induced secondary osteopenia in female rats: a time course study as compared with ovariectomy-induced osteopenia and response to salmon calcitonin. Jpn. J. Pharmacol., 79, 379-386.

Nozaka, K., Miyakoshi, N., Kasukawa, Y., Maekawa, S., Noguchi, H. \& Shimada, Y. (2008) Intermittent administration of 
human parathyroid hormone enhances bone formation and union at the site of cancellous bone osteotomy in normal and ovariectomized rats. Bone, 42, 90-97.

Orloff, J.J., Goumas, D., Wu, T.L. \& Stewart, A.F. (1991) Interspecies comparison of renal cortical receptors for parathyroid hormone and parathyroid hormone-related protein. J. Bone Miner. Res., 6, 279-287.

Parfitt, A.M., Drezner, M.K., Glorieux, F.H., Kanis, J.A., Malluche, H., Meunier, P.J., Ott, S.M. \& Recker, R.R. (1987) Bone histomorphometry: standardization of nomenclature, symbols, and units. Report of the ASBMR Histomorphometry Nomenclature Committee. J. Bone Miner. Res., 2, 595-610.

Petersen, M.M., Gehrchen, P.M., Nielsen, P.K. \& Lund, B. (1997) Loss of bone mineral of the hip assessed by DEXA following tibial shaft fractures. Bone, 20, 491-495.

Qin, L., In, P., Wang, L., Li, X., Swarthout, J.T., Soteropoulos, P., Tolias, P. \& Partridge, N.C. (2003) Gene expression profiles and transcription factors involved in parathyroid hormone signaling in osteoblasts revealed by microarray and bioinformatics. J. Biol. Chem., 278, 19723-19731.

Seto, H., Aoki, K., Kasugai, S. \& Ohya, K. (1999) Trabecular bone turnover, bone marrow cell development, and expression of bone matrix proteins after low calcium feeding in rats. Bone, 25, 687-695.

Skripitz, R. \& Aspenberg, P. (2001) Early effects of parathyroid hormone (1-34) on implant fixation. Clin. Orthop. Relat. Res., 392, 427-432.

Sutherland, M.K., Geoghegan, J.C., Yu, C., Turcott, E., Skonier, J.E., Winkler, D.G. \& Latham, J.A. (2004) Sclerostin promotes the apoptosis of human osteoblastic cells: a novel regulation of bone formation. Bone, 35, 828-835.
Tamura, Y., Miyakoshi, N., Itoi, E., Abe, T., Kudo, T., Tsuchida, T., Kasukawa, Y. \& Sato, K. (2001) Long-term effects of withdrawal of bisphosphonate incadronate disodium (YM175) on bone mineral density, mass, structure, and turnover in the lumber vertebrae of ovariectomized rats. J. Bone Miner. Res., 16, 541-549.

Tintut, Y., Parhami, F., Le, V., Karsenty, G. \& Demer, L.L. (1999) Inhibition of osteoblast-specific transcription factor Cbfal by the cAMP pathway in osteoblastic cells. J. Biol. Chem., 274, 28875-28879.

Tsuchida, T., Miyakoshi, N., Kudo, T., Tamura, Y., Kasukawa, Y., Suzuki, K. \& Sato, K. (2001) Restoring effects of human parathyroid hormone (1-34) on trabecular connectivity in ovariectomized rats. Tohoku J. Exp. Med., 194, 213-221.

Tsuchida, T., Sato, K., Miyakoshi, N., Abe, T., Kudo, T., Tamura, Y., Kasukawa, Y. \& Suzuki, K. (2000) Histomorphometric evaluation of the recovering effects of human parathyroid hormone (1-34) on bone structure and turnover in streptozotocin-induced diabetic rats. Calcif. Tissue Int., 66, 229-233.

Tsuchie, H., Miyakoshi, N., Kasukawa, Y., Aonuma, H. \& Shimada, Y. (2011) The effects of circumferential wiring on cancellous bone healing after osteotomy in ovariectomized rats. Akita J. Med., 38, 21-26.

Wenzl, M.E., Porte, T., Fuchs, S., Faschingbauer, M. \& Jurgens, C. (2004) Delayed and non-union of the humeral diaphysiscompression plate or internal plate fixation? Injury, 35, 55-60.

Zhang, X., Schwart, E.M., Young, D.A., Puzas, J.E., Rosier, R.N. \& O'Keefe, R.J. (2002) Cyclooxygenase-2 regulates mesenchymal cell differentiation into the osteoblast lineage and is critically involved in bone repair. J. Clin. Invest., 109, 14051415. 